\title{
Standounkt
}

Zur Unwirksamkeit marktbasierter Instrumente im Umweltschutz

\section{Trügerische Konzepte und ökonomische Verrenkungen}

Von profitorientierter Wirtschaft verursachte Natur- und Umweltzerstörung mit marktwirtschaftlichen Mechanismen begrenzen zu wollen, stellt einen logischen Kurzschluss dar. Doch warum lassen sich Naturschutzorganisationen darauf ein? Von Klaus Pedersen

D e Natur schrumpft, und aus sehr unterschiedlichen Motiven bemüht sich eine Minorität der Weltgesellschaft, dieser Entwicklung Einhalt zu gebieten.

Till Pistorius von der Universität Freiburg dürfte den Teilnehmern der Vorbereitungskonferenz deutscher Nichtregierungsorganisationen auf die Vertragsstaatenkonferenz zur UN-Konvention über biologische Vielfalt (COP9) aus dem Herzen gesprochen haben, als er mehr finanzielle Mittel für Waldschutzgebiete (WSG) forderte. Gebraucht wird das Geld in den Ländern des Südens, um Finanzierungslücken bei bestehenden WSG zu schließen beziehungsweise diese zu erweitern und um neue WSG auszuweisen (Pistorius 2007).

\section{Ökonomische Verrenkungen}

Angesichts der von der Rezession gestressten Staatshaushalte dürfte sein Vorschlag, diese Mittel durch die Vermarktung von Ökosystemleistungen zu beschaffen, noch aktueller geworden sein. Zunächst soll es eine Fokussierung auf Kohlenstoffmärkte geben, aber auch Bioprospektion und Ökotourismus werden als Finanzierungsquellen betrachtet. Mit diesem Lösungsansatz will man jene Natur- und Umweltzerstörung, die maßgeblich von profitorientierter Wirtschaft verursacht wird, mit marktwirtschaftlichen Mechanismen begrenzen.

Bei den auf diese Weise finanzierten Schutzgebieten werden die massiven Menschenrechtverletzungen ausgeblen- det, zu denen es in der Vergangenheit immer wieder gekommen ist (Pedersen 2008).

Doch fokussieren wir uns auf die Kohlenstoffmärkte: „(G)emäß dem seit 2005 geltenden Kyoto Protokoll können bislang nur leistungsbasierende Kohlenstoffdioxid-Kredite gehandelt werden (Aufforstungen etc.), während die Besitzer von stehenden Wäldern leer ausgehen“, schreibt Schmidt-Soltau (2008). Er verweist damit darauf, dass der Handel mit Kohlenstoffdioxid-Krediten ein hoch spekulativer Markt ist, bei dem „niemand so genau weiß, was der Wert der Ware ist." Dabei stellt er den gewaltigen gesellschaftlichen Gebrauchswert der Regenwälder in Kontrast zu der unsinnigen Praxis, vor allem für Aufforstungen Kohlenstoffdioxid-Kredite $\mathrm{zu}$ vergeben (Stern 2007).

Doch die ökonomischen Verrenkungen hören dort nicht auf. Der Gesamtertrag einer Ölpalmplantage beträgt 347 Euro pro Hektar und Jahr, während sich die Gesamtsumme der für den Naturschutz gebotenen Ausgleichszahlungen auf 1,15 Euro pro Hektar und Jahr beläuft (Schmidt-Soltau 2008). Das klingt nach dem Wettlauf zwischen Hase und Igel.

Den Hasen stellt im vorliegenden Fall der World Wide Fund for Nature (WWF), der mit seiner Präsenz am konzerndominierten Round Table for Sustainable Palmoil diesen quasi grünwäscht, denn der Öffentlichkeit wird immer wieder suggeriert, er sei eine Art Zertifizierungsinstitution. Dieses Beispiel illustriert die Spiel-

regeln und das Kräfteverhältnis im globalen Ringen um Ressourcen und Umweltschutz. Was treibt die großen Naturschutzorganisationen dazu, sich auf dieses Spiel einzulassen statt die Spielregeln öffentlich zu hinterfragen?

\section{Subsistenz auf hohem Niveau}

In einer Welt endlicher Ressourcen sollte die Einsicht nahe liegend sein, dass eine auf Wachstum basierende Wirtschaft langfristig in die Sackgasse führt. Versuche, das Tempo dahingehend durch Ausgleichsmaßnahmen zu reduzieren, sind unter Umständen löblich.

Doch parallel dazu wäre die vehemente Suche nach umfassenden, gesamtgesellschaftlichen Lösungen prinzipieller Art erforderlich. Wenn sich die Anstrengungen in technokratischen Lösungsvorschlägen für Teilprobleme erschöpfen, die Aufschub versprechen und zugleich die Partikularinteressen der Mächtigen bedienen, dürfte uns das Ende der Sackgasse kaum erspart bleiben. Wo bleibt das Konzept für eine lebenswerte Gesellschaft ohne Wachstum, für eine Subsistenzwirtschaft auf hohem Niveau?

\section{Literatur}

Pedersen, K.: Naturschutz und Profit - Menschen zwischen Vertreibung und Naturzerstörung. Münster 2008.

Pistorius, T.: Finanzierungs- und Umsetzungsmechanismen für ein globales Waldschutzgebiets-Netzwerk unter der CBD. Präsentation auf der Vorbereitungskonferenz der deutschen NGOs für die COP9 der CBD, 11./12.12.2007.

Schmidt-Soltau, K.: Gas, Preis und Profit. Eine kleine Ökonomie des $\mathrm{CO}_{2}$-Handels. In: Zeitschrift Marxistische Erneuerung 76, 2008 S. 143-152.

Stern, N.: The Economics of Climate Change: The Stern Review. Cambridge 2007.

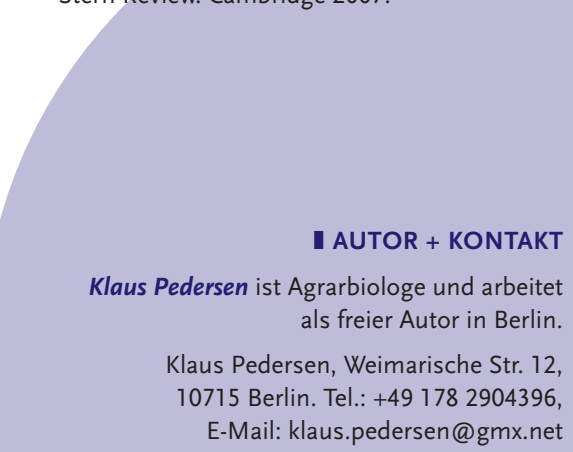


(c) 20I0 Authors; licensee IÖW and oekom verlag. This is an article distributed under the terms of the Creative Commons Attribution Non-Commercial No Derivates License (http://creativecommons.org/licenses/by-nc-nd/3.o/), which permits unrestricted use, distribution, and reproduction in any medium, provided the original work is properly cited. 